\title{
Rhetorice contracta (1621), II, 22, « Les discours de sédition et d'union "
}

Notes et édition du texte Francis Goyet, traduction Laurence Vianès

\section{G. J. Vossius}

Francis Goyet (éd.)

Traducteur : Laurence Vianès

\section{OpenEdition \\ Journals}

Édition électronique

URL : http://journals.openedition.org/rhetorique/170

DOI : $10.4000 /$ rhetorique. 170

ISSN : 2270-6909

Éditeur

UGA Éditions/Université Grenoble Alpes

Édition imprimée

ISBN : 978-2-84310-255-4

Référence électronique

G. J. Vossius, «Rhetorice contracta (1621), II, 22, «Les discours de sédition et d'union » », Exercices de rhétorique [En ligne], 1 | 2013, mis en ligne le 12 novembre 2013, consulté le 12 septembre 2020. URL http://journals.openedition.org/rhetorique/170 ; DOI : https://doi.org/10.4000/rhetorique.170

Ce document a été généré automatiquement le 12 septembre 2020.

\section{(c) (i) (9)}

Les contenus de la revue Exercices de rhétorique sont mis à disposition selon les termes de la Licence Creative Commons Attribution - Pas d'Utilisation Commerciale - Partage dans les Mêmes Conditions 4.0 International. 


\title{
Rhetorice contracta (1621), II, 22, «Les discours de sédition et d'union »
}

Notes et édition du texte Francis Goyet, traduction Laurence Vianès

\section{G. J. Vossius}

Francis Goyet (éd.)

Traduction : Laurence Vianès

\begin{abstract}
Texte de l'éd. de 1660 : Rhetorices contractae, sive Partitionum Oratoriarum libri quinque, Leipzig, Christian Kirchner, II, 22, p. 190-194 ; $1^{\mathrm{e}}$ éd. Leyde, J. Maire, 1621 ; nombreuses rééd. jusqu'à 1698, dont certaines sur Google (qui a aussi l'éd. Madrid, Anton. Sancha, 1781). Rhetorices, en caractères latins, est le mot grec rhètorikè au génitif, litt. « les cinq livres de la Rhétorique abrégée ». Le titre Partitiones reprend celui de Cicéron, Partitiones Oratoriae, les Divisions de l'art oratoire : la rhétorique classée, pour les élèves.
\end{abstract}

Mêmes principes d'édition que précédemment; dans le latin, nous ajoutons les guillemets.

1 Chap. XXII. Les discours de sédition et d'union. DE CONCITATIONE, ET CONCILIATIONE.

\section{$\S 1$.}

2 Voyons maintenant la technique pour faire des discours de sédition et d'union ${ }^{1}$. Quant au premier type de discours, de même qu'il mérite rarement l'éloge, de même il ne demande pas non plus beaucoup d'art : surtout si nous avons affaire à une foule inculte. Qui en effet n'est pas bouleversé au plus haut point quand il s'aperçoit qu'on commet une injustice épouvantable envers lui ou les siens? Il suffira donc ici de ce que nous avons dit plus haut, dans les chapitres sur les passions de la colère et de la haine. 
Nunc videamus de concitandi et conciliandi artificio. Ac prius quidem illud, ut raro laudem meretur, ita nec multum artis requirit: praesertim si negotium sit cum multitudine imperita. Nam quotusquisque non maximopere commovetur, si sibi, vel suis, atrocem fieri injuriam intelligat? Quare hic sola suffecerint, quae de irae et odii affectibus supra diximus.

J'en donnerai seulement un exemple tiré d'une autorité incontestable, Tacite ${ }^{2}:$ « [An. I, 16, 3] Il y avait dans le camp un certain Percennius, autrefois chef d'entreprises théâtrales, depuis simple soldat, parleur audacieux, et instruit, parmi les cabales des histrions, à former des intrigues ${ }^{3}$. Comme il voyait ces esprits incultes ${ }^{4}$ en peine de ce que serait après Auguste la condition des gens de guerre, il les ébranlait peu à peu dans des entretiens nocturnes; ou bien, sur le soir, lorsque les hommes tranquilles étaient retirés, il assemblait autour de lui tous les pervers ${ }^{5}$. [An. I, 17, 1] Enfin, lorsqu'il se fut associé de nouveaux artisans de sédition, prenant le ton d'un général qui harangue, il demandait aux soldats: "POURQUoI obéissaient-ils en esclaves à un petit nombre de centurions, à un petit nombre de tribuns? Quand donc oseraient-ils réclamer du soulagement, s'ils n'essayaient, avec un prince nouveau et chancelant encore, les prières ou les armes? [An. I, 17, 2] C'était une assez longue et assez honteuse lâcheté, de courber, trente ou quarante ans, sous le poids du service <militaire>, des corps usés par l'âge ou mutilés par les blessures." » Et un peu plus loin [An. I, 17, 4] : "Le service en lui-même était pénible, infructueux : dix as par jour, voilà le prix qu'on estimait l'âme et le corps du soldat ; là-dessus, il devait se fournir d'armes, d'habits, de tentes, se racheter de la cruauté des centurions, payer les moindres dispenses ${ }^{6}$. Mais les verges, mais les blessures, de rigoureux hivers, des étés laborieux, des guerres sanglantes, des paix stériles, à cela jamais de fin" ", etc. À cette assemblée $e^{7}$ prête à la sédition, ce qui mit le feu, ce fut le discours de Vibulenus, rapporté peu après ${ }^{8}$. Les procédés de ce genre de style virulent, procédés ou pour mieux dire poisons ${ }^{9}$, il convient de les connaître non pas pour s'en servir, mais pour mieux s'en protéger.

Exemplum tantummodo adferam ex gravissimo scriptore, Tacito ${ }^{10}$ : [An. I, 16, 3] «Erat », inquit, « in castris Percennius quidam, dux olim theatralium operarum, dein gregarius miles, procax lingua, et miscere coetus histrionali studio doctus. Is imperitos animos, et, quaenam post Augustum militiae conditio, ambigentes, impellere paullatim nocturnis colloquiis, aut flexo in vesperam die, et, dilapsis melioribus, deterrimum quemque congregare: [An. I, 17, 1] postremo, promptis jam et aliis seditionis ministris, velut concionabundus, interrogabat: CUR PAUCIS centurionibus, paucioribus tribunis, in modum servorum obedirent? quando ausuros exposcere remedia, nisi novum, et nutantem adhuc principem, precibus, vel armis, adirent? [An. I, 17, 2] satis per tot annos ignavia peccatum, quod tricena, et quadragena stipendia senes, et plerique truncato [p. 191] ex vulneribus corpore, tolerent. » Et mox [An. I, 17, 4]: «Enimvero <militiam ipsam> gravem, infructuosam, denis in diem assibus, animam et corpus aestimari. hinc vestem, arma, tentoria, hinc saevitiam centurionum, et vacationem munerum redimi. at hercle verbera et vulnera, duram hiemem, exercitas aestates, bellum atrox, aut sterilem pacem, sempiterna, " etc. Seditiosae isti concioni faces subdidit oratio Vibuleni, quam paullo post refert. Hujusmodi autem virulentae linguae artificia, seu veneficia malis, nosse convenit, non ut utamur, sed ut melius ab iis caveamus nobis.

\section{$\S 2$.}

4 À la sédition s'oppose l'UNION, que nous pouvons considérer comme double, car soit nous nous concilions l'amitié et alliance de quelqu'un, soit nous réconcilions des partis qui s'affrontent. 
Concitationi opponitur CONCILIATIO. Quae bifariam sit. nam vel nobis amorem alicujus conciliamus : vel in gratiam reducimus partes dissidentes.

\section{§ 3. [Conciliatio 1 : amitié et alliance]}

5 Dans la première catégorie, voici les deux parties de notre discours. Premièrement nous exposons à quel titre et pour quels motifs nous nous enhardissons à solliciter la bienveillance et la faveur de quelqu'un ${ }^{11}$. C'est ici que trouve place l'éloge de celui que nous souhaitons avoir pour ami. Cependant il faut prendre garde à ce pour quoi chacun veut être estimé principalement. Car l'éloge de la générosité est banal, on peut l'adresser quasiment à tout le monde ${ }^{12}$. En revanche, d'autres éloges sont plus le propre de chacun. Par exemple, un philosophe se plait à s'entendre louer pour l'acuité de son intelligence et l'ampleur de ses connaissances; un sénateur, pour son exceptionnelle clairvoyance et son dévouement à ses concitoyens ; un général, pour sa vaillance et ses hauts faits. Autre point : l'éloge d'un jeune homme pourra se faire franchement, celui d'un homme plus âgé devra faire preuve d'habileté. Dans un cas comme dans l'autre, nous nous mettrons autant que possible à l'abri du soupçon de flatterie ${ }^{13}$.

In priori duo haec praestamus. Primum exponimus, qua fiducia, et quibus de causis, alicujus benevolentiam, et favorem ambiamus. Ubi laus ejus, quem amicum volumus, habet locum. Spectandum autem, qua quisque re imprimis velit aestimari. Humanitatis laus quasi communis omnium videtur. At aliae magis sunt propriae. Ut philosophus gaudet laudari ab ingenio perspicaci, et multarum disciplinarum cognitione: senator a prudentia singulari, et benevolentia erga cives: imperator a fortitudine, et rebus gestis. Ac [p. 192] juvenem quidem laudare licebit apertius: in sene id faciemus cautius. In utrisque adulationis suspicionem, quantum licet, amovebimus.

\section{$\S 4$.}

6 La deuxième partie de notre discours montrera que nous ne sommes pas indignes de la faveur que nous sollicitons : s'allier avec nous ne sera ni inutile ni déshonorant ${ }^{14}$. Aussi nous ferons ici l'éloge de nous-mêmes, mais il y faut mettre la clairvoyance nécessaire, pour ne pas trop avoir l'air de claironner son propre éloge.

Pars altera ostendet, favore non esse nos indignos : amicitiam nostram nec inutilem fore, nec indecoram. Quare hic nosmetipsos laudabimus. sed ea opus est prudentia, ut nequis laudis suae nimis videatur buccinator.

7 Voyez la lettre de Cicéron à Métellus Népos, Familiares V, IV ; également la lettre V, IX, de Vatinius à Cicéron ${ }^{15}$. On trouve un exemple particulièrement magnifique de ce procédé chez un auteur récent mais fort distingué, Ange Politien, à l'ouverture de sa lettre à Matthias roi de Pannonie, laquelle est la première du livre IX.

Vide epistolam Ciceronis ad Metellum Nepotem, quae est quarta libri quincti. item nonam ejusdem libri, quae Vatinii est ad Ciceronem. At luculente imprimis hoc artificium aperit illa recentis, sed politissimi scriptoris, Angeli inquam Politiani epistola ad Matthiam, Pannoniae regem, quae prima est libri noni.

\section{§ 5. [Conciliatio 2 : réconciliation]}

8 Pour réconcilier des partis qui s'affrontent, voici comment procéder. Nous montrons que toute offense doit être effacée : d'une part parce que c'est plus utile et plus sûr, et 
d'autre part, parce que c'est aussi plus honorable ${ }^{16}$. Les passions qui règnent ici sont l'espoir et la crainte ${ }^{17}$.

Partes dissidentes conciliamus hoc pacto. Ostendimus, offensam omnem obliterari debere: partim quia utilius tutiusque est : partim etiam quia honestius. Ex affectibus hic regnant spes et metus.

\section{$\S 6$.}

9 Mais autant il est facile d'exciter les gens à la sédition, autant il est difficile de les réconcilier. Car la plupart des gens sont trop attachés à leur colère et au souvenir de l'offense reçue. Là où il est le plus facile de ramener l'union, c'est face à une foule excitée $^{18}$. Alors peu importe que le discours soit éloquent, ce qui compte pour l'orateur est la protection d'un double bouclier ${ }^{19}$, à savoir sa popularité et sa supériorité hiérarchique.

Quemadmodum vero concitare homines facile est : ita difficile est eosdem conciliare. Sunt enim plerique omnes irae, atque offensae, nimis tenaces. Ac nusquam facilius opera luditur, quam apud [p. 193] multitudinem concitatam. Ubi haut multum valet diserta oratio, nisi eam duplex muniat praesidium, benevolentia popularis, et dicentis majestas.

D'où ces vers de Virgile, après avoir décrit une sédition populaire :

Si alors un homme, dont la piété, dont les mérites justifient l'autorité,

se présente à leurs yeux, on se tait, on l'écoute.

De fait, Jules César calma son armée en appelant ses hommes «Citoyens!» au lieu de "Soldats !20 »; et par ce seul mot, il leur fit faire volte-face et changer d'humeur, si bien qu'ils répondirent qu'ils étaient des soldats, et quoiqu'il leur contestât ce nom, ils le suivirent plus loin en Afrique. On voit aussi chez Tacite combien peu de mots il fallut au centurion Clément pour calmer la sédition provoquée par Percennius et Vibulenus, dont nous avons parlé plus haut. Le point est que, comme le dit l'illustre historien, «ses bons procédés envers eux le rendaient agréable aux soldats ${ }^{21}$ ». D’ailleurs, il avait eu lui aussi une part de responsabilité dans la sédition; mais il était revenu à de meilleurs sentiments.

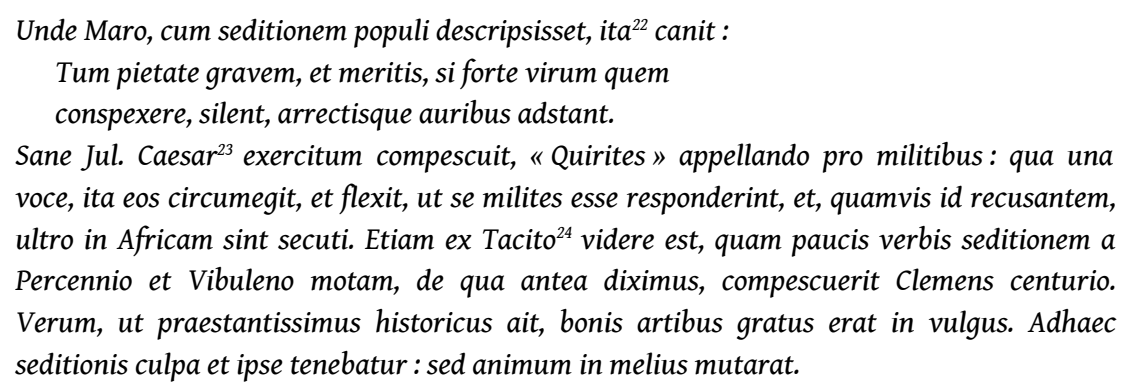

\section{$\S 7$.}

11 Cela posé, attention: il est très important que personne ne se risque à faire face immédiatement à une foule aveuglée par la colère, en s'imaginant protégé par son intégrité et son autoritée 25 Car le premier mouvement d'une foule étant tout simplement belliqueux, ce n'est pas un discours qui vous permet de reprendre la main. En revanche, quand l'effervescence sera retombée, ce sera le moment propice pour leur parler. Mais d'abord il faudra établir le diagnostic sur l'origine de ce grand accès de fureur. Car le médecin qui ignore où est la plaie, comment y portera-t-il remède ? Le 
remède lui-même devra garder un certain équilibre : le discours commencera sur un ton amical, calmant ; de là, nous passerons à des paroles de réprimande, plus sévères. Et dans l'application de l'un comme de l'autre remède, on respectera le dosage qu'exige la situation.

Porro multum etiam refert, ut nequis efferatae plebi statim se credat, utcumque et innocentiae, et autoritatis praesidio tutus sibi videatur. Nam primus ille multitudinis impetus plane est belluinus, ut oratione regi non possit. At ubi is deferbuit, idoneum magis orandi tempus erit. Sed prius constare oportebit, quae origo sit mali tanti. Nam qui ignorat, qua parte sit vulnus, quomodo ei faciet medicinam? Etiam temperamento istoc in oratione opus erit, ut prius amice deliniamus; hinc verbis castigemus severius. Atque interim modus ille in utroque hoc remedio tenendus, quem res, et tempus postulabunt.

César Germanicus ne l'a pas ignoré. Pour calmer une révolte qui s'était déclarée, et comme le raconte Tacite $[A n . \mathrm{I}, 34,4]$, « il commença par rendre un pieux hommage à Auguste, puis il passa aux victoires et aux triomphes de Tibère, en exaltant spécialement ses glorieuses campagnes en Germanie à la tête de ces légions-là. Ensuite il exalta l'unanimité de l'Italie, la fidélité des Gaules, l'absence partout de troubles ou de désaccords. » Et puis, de là, insensiblement ${ }^{26}$, il arriva à ces mots $[A n . \mathrm{I}, 35,1]:$ « Où est la subordination militaire? Où est la discipline qui vous faisait honneur autrefois? Qu'avez-vous fait de vos tribuns, de vos centurions?» Cependant ce n'est pas avec ce discours qu'il parvint vraiment à les faire changer d'humeur ${ }^{27}$; mais ce que ni son éloquence d'homme ni son autorité n'avaient pu obtenir, se produisit avec le spectacle de son épouse, portant sur son sein leur fils encore tout petit, accompagnée des seules épouses de ses amis, quand en un cortège pitoyable toutes se réfugièrent chez les Trévires: le sens de l'honneur et la pitié, d'une part, et d'autre part la jalousie envers les Trévires, voilà ce qui arracha le revirement des soldats ${ }^{28}$.

Non ignoravit hoc Caesar Germanicus, qui ut obortam seditionem sopiret, "a veneratione Augusti orsus," (quemadmodum Tacitus ${ }^{29}$ narrat [An. I, 34, 4]) "flexit ad victorias, triumphosque Tiberii; praecipuis laudibus celebrans, quae apud Germanias illis cum legionibus pulcherrimas fecisset? Italiae inde consensum, Galliarum fidem extollit, nil usquam turbidum, aut discors. " Atque ab his sensim ad illa verba perductus est [An. I, 35, 1]: «Ubi modestia militaris? ubi veteris disciplinae decus? quonam tribunos? quonam centuriones exegistis?" Quamquam parum quidem oratione ea flexit: sed, quod nec facundia viri, nec autoritas potuit, id, cum ducis uxor, parvulum sinu filium gestans, et una amicorum uxores, miserabili agmine ad Treviros fugerent; partim pudor et miseratio, partim invidia in Treviros, extorsit.

\section{NOTES}

1. Discours de sédition et d'union : ce deuxième descriptif introduit une seconde innovation. Vossius a d'abord ajouté à Junius (et donc à Érasme) les discours de réconciliation trouvés chez les historiens. Il ajoute maintenant à cette conciliatio 2 son pendant chez les mêmes historiens, le discours qui appelle à la sédition, qu'il baptise concitatio avec une belle paronomase, même si seditiosus vient rapidement sous sa plume. Font irruption sur scène le bruit et la fureur de la guerre, et surtout de la guerre civile. L'historien de référence n'est plus Tite-Live mais Tacite, Vossius méditant le début des Annales. On s'éloigne ainsi de plus en plus de l'irénisme d'Érasme. 
Concitatio est litt. le fait d'exciter (concitare), de soulever toute sorte de passions, avec comme sens spécialisé « soulèvement », insurrection : chez Cicéron, Brutus, 56, « concitatio et seditio » n'est pas un doublon mais une progression, la plèbe est d'abord excitée à la sédition puis elle fait sécession d'avec les sénateurs. Conciliatio est facile à traduire dans le cas particulier où il s'agit de renouer après une sédition (conciliatio 2). Il est plus difficile de trouver un terme qui englobe les conciliatio 1 et 2 . Nous nous autorisons de la trad. du jésuite François Pomey : « union et amitié des uns avec les autres » (Syllabus seu Lexicon latino-gallico-graecum, Lyon, M. Mauteville, 1757, Google ; avec conciliatricula, « celle qui s'entremet, qui fait la paix »). C'est la « pacification », mais au sens des édits de pacification entre catholiques et protestants (Littré, pacification, $2^{\mathrm{e}}$, " apaisement des dissensions domestiques, des différends entre particuliers"; voir n. 1 du premier document, «apaiser, réconcilier» pour epareskomai). Après le discours de guerre civile ou concitatio, le discours de paix ; après l'embrasement, l'apaisement. On est proche du couple ira et lenitas, colère et retour au calme (voir au $\S 7$ deliniamus, sur delenio et donc lenis). De ce point de vue, la fameuse captatio benevolentiae signifie en somme "nous venons en paix»: voir le doublon "pax et benevolentia » chez Cicéron, « paix et amitié » (et non " sérénité et bienveillance », trad. G. Achard dans son éd. du De inventione, à I, 21, Paris, Les Belles Lettres, 1994).

2. Trad. J. L. Burnouf, Paris, Hachette, 1859 (sur Itinera Electronica).

3. Intrigues: dans la trad. d'Ablancourt, "autrefois chef de faction dans les Théâtres, soldat insolent et expert aux séditions populaires » (Les CEuvres de Tacite, Amsterdam, Ravesteyn, 1670, t. I, p. 16 ; Google).

4. Incultes: pour imperitos (« simples», Burnouf; esprits « légers », d'Ablancourt). Voir le imperita juste avant, " une foule inculte ». C'est l'antonyme de peritus, « habile, qui sait par expérience » (Gaffiot).

5. Tous les pervers: dans la trad. Paul Mattéi (sur BLL), «tous les plus mauvais sujets » face aux « meilleurs éléments".

6. Dispenses : Vossius écrit vacationem, là où toutes les éd. de Tacite ont vacationes.

7. Assemblée : concio, qui reprend le concionabundus de Tacite au début de I, 17, 1, «prenant le ton d'un général qui harangue ». La concio ou contio est à la fois (Gaffiot) «l'assemblée du peuple convoquée et présidée par un magistrat» et "le discours public» tenu devant une telle assemblée; à l'armée, cela devient «l'assemblée des soldats» et «la harangue tenue aux troupes ».

8. Le discours de Vibulenus, rapporté peu après : à An. I, 22. Vibulenus, simple soldat, enflamme la foule en prétendant mensongèrement que son propre frère a été égorgé par les gladiateurs aux ordres du général ou « légat » Blaesus. Après le discours de Percennius, Blaesus a tenté d'apaiser la sédition, mais celle-ci a repris de plus belle (I, 21). La formule de Vossius, faces subdidit, litt. «mettre les torches sous (le bûcher) ", évoque la première phrase de Tacite à I, 22, 1, Flagrantior inde vis, métaphore « rare et hardie » selon Pierre Wuilleumier (Tacite, Annales, Paris, Les Belles Lettres, 1978), lequel traduit «Alors l'embrasement redouble de violence » (d'Ablancourt : «La sédition s'échauffe »). Les mots mêmes de Vossius sont chez Quintilien (I, 2, 25) : «Id nobis acriores ad studia dicendi faces subdidisse quam exhortationem docentium, Cette pratique [le concours d'éloquence entre élèves] nous enflammait encore plus pour l'étude de la parole que les exhortations de nos maîtres ». Les exhortations ont « chauffé » le bois du bûcher, il ne reste plus qu'à introduire dessous (sub) les torches ou brandons (fax, pl. faces). Cf. l'image moderne du feu aux poudres. Le discours de Percennius rassemble et sèche la poudre, le discours suivant y met le feu.

9. Procédés... poisons: le latin fait rimer artificia et veneficia, «artifices ou pour mieux dire maléfices » (veneficium, poison, philtre magique ou sortilège).

10. «Lib. 1. Annalium. » (note de Vossius)

11. Solliciter la bienveillance et la faveur : reprise du tout début de Junius sur la conciliatoria. Scholae, p. 113 : « in quibus favorem ac benevolentiam conciliare nobis eorum studemus », puis on demande au 
destinataire aide et protection, comme le cliens à un patronus : "vel in studiis, vel rebus aliis juvare atque promovere ".

12. Quasiment à tout le monde : litt. l'éloge de la générosité " est pour ainsi dire commun à tous ». C'est un défaut majeur : le destinataire veut être distingué d'avec les autres et non pas confondu avec eux, voir Aristote (Rhétorique, II, 2, 1378b30 : si on oublie l'éloge d'une qualité particulière, on suscite la colère de celui à qui on s'adresse, en ne l'estimant pas par où il s'estime devoir l'être). Vossius critique ici Junius, qui ne voit pas le problème. Celui-ci (p. 114) met au plus haut la demande adressée par un jeune homme à un lettré ou savant. Selon lui, plus on est doctus, plus on est aussi humanus, c'est-à-dire prêt à aider généreusement : "Quo enim quisque est doctior : eo et humanior esse, ac laetari magis solet occasionem sibi benefaciendi aliis offerri ». À défaut, ou en second lieu, quand il faut s'adresser aux non lettrés (indocti), alors on en viendra à l'éloge adapté à chacun. Junius cite le général puis le sénateur, et retombe pour finir (p.115) sur l'humanitas commune à tous: "Omnes tamen fere humanitatis delectari laude videntur: neque quisquam liberali erectoque est proditus ingenio, qui non inde commendari cupiat." L'humanitas est, à suivre Hellegouarc'h (p. 267-271), la qualité même d'un protecteur ou patronus. S'y trouvent « englobées toutes les formes du comportement de l'homme d'État romain : d'une part son attitude envers ceux dont il doit solliciter les faveurs et sur lesquels il doit appuyer son action politique ; mais aussi la situation d'un homme de classe supérieure qui s'impose aux autres par une attitude sage et modérée que lui inspirent son éducation et sa culture. » L'humanitas s'applique « aux relations entre amis ", non seulement "politesse » mais, de façon plus large, "attitude obligeante et courtoise de quelqu'un qui s'efforce de rendre service » : « ni "humanisme" ni "humanitarisme", l'humanitas consiste alors simplement à remplir les devoirs de l'officium. "

13. Soupçon de flatterie: "suspicionem assentationis» (Érasme, LB I, 478), "adulationis suspicio » (Junius, p. 116). Vossius ajoute à Junius le distinguo entre éloge d'un jeune homme et d'un plus âgé. La formule "dans un cas comme dans l'autre " renvoie soit à ces deux âges; soit plus sûrement aux deux défauts ou vitia de l'éloge décrits dans ce paragraphe, a) être banal, b) manquer de retenue.

14. Ni inutile ni déshonorant: ce sont les deux lieux du délibératif, "l'utile et l'honnête ", les intérêts bien compris et l'honorable, lequel est soit le beau moral, soit (à défaut selon Junius) la gloire. En effet, Junius (p. 116-117) attribue le goût de l'utile et le désir de gloire aux seuls indocti : "Sed et movendus erit animus ejus ad quem scribimus, maxime spe ac fiducia : et promittenda, literatis ac prudentibus laus, jucunditas honesta, praedicatio perpetua : indoctis utilitas et commoda quibus magis illi quam honestate, aut [p. 117] laude atque gloria moveri solent. » - À noter que Vossius ne reprend pas à Junius les passions à susciter, « surtout espoir et assurance » selon ce dernier.

15. Familiares, V, 4 ( «Litterae Q. fratris») et V, 9 ("Si tuam consuetudinem»): Cicéron, Correspondance, Paris, Les Belles Lettres, t. II (éd. L.-A. Constans, 1941) et t. VIII (éd. J. Beaujeu, 1983), lettres $n^{\circ} 88$ et 682 . Ce sont les deux premiers exemples de Junius. À V, 4, 2, Cicéron évoque le discours très modéré de son destinataire et adversaire acharné Métellus Népos, lors de la séance solennelle du Sénat un mois auparavant, le $1^{\mathrm{er}}$ janvier 57 (voir la note de Constans, t. II, p. 80). Pour Melanchthon, ces deux lettres sont simplement une demande, petitio; il qualifie de conciliatio une lettre plus longue et plus claire, Fam. III, 9.

16. Plus utile... plus honorable: même couple de «l'utile et l'honnête " qu'au §4. Mais cette fois, Vossius précise les passions qui s'y raccrochent. Oublier les offenses est préférable parce que plus conforme a) à nos intérêts et à notre sécurité (passion : crainte, avec son inverse, l'espoir) ; b) au beau moral.

17. L'espoir et la crainte: repris de la «didascalie» qui chez Tacite précède le petit discours d'apaisement tenu par le centurion Clément (nommé au § 6) et quelques autres. «Spem offerunt, metum intendunt », « ils présentent l'espoir, brandissent la peur » (An. I, 28, 4, trad. Wuilleumier), ou, chez d'Ablancourt, « réveillent l'espérance du pardon, et la crainte du châtiment » (p. 24 ; Google). 
18. Facile... face à une foule excitée : c'est relativement plus facile, la foule étant dans l'immédiateté, quand le Roi ou un Grand dans sa colère n'oublie jamais une offense. Voir l'histoire de Fargues, décapité après avoir été retrouvé en forêt lors d'une chasse (Saint-Simon, Mémoires, année 1705, ch. XXX).

19. Double bouclier: nous transposons l'image de praesidium, "garde, escorte » (Gaffiot). Dans la Rome antique comme au XVII ${ }^{\mathrm{e}}$ siècle, un grand personnage ne se déplace jamais sans une garde rapprochée, sans un entourage important. C'est ici une double garde: les bonnes dispositions (benevolentia) du peuple envers l'orateur; la «majesté » de ce dernier. Machiavel ne parle que de la seconde ou autorité (Discours sur la première décade de Tite-Live, I, 54), en citant les mêmes vers de Virgile, célébrissimes (Aen. I, 151-152), quand l'apparition de Neptune suffit à calmer la tempête ou sédition des vents. Pour ces vers, nous nous inspirons de la trad. Desfontaines, Paris, Quillau, 1743 : «Si alors un homme responsable se présente à leurs yeux, on se tait, on l'écoute ». Trad. J. Perret (Paris, Les Belles Lettres, 1977) : «alors s'ils ont vu un homme que sa piété, ses œuvres rendent vénérable, ils font silence, s'immobilisent, l'oreille dressée »; ensuite, «il parle, et sa parole gouverne les cœurs, calme les passions » (Desfontaines : « et bientôt la sédition est apaisée »).

20. Soldats!: Vossius reprend la formulation de Germanicus, un peu plus loin dans Tacite (An. I, 42, 3 ; cf. Suétone, Cés. 70, et Dion, XLII, 53, 3), « seditionem exercitus verbo uno compescuit, Quirites vocando qui sacramentum ejus detrectabant ", "César arrêta d'une seule parole ses soldats, en les appelant "bourgeois", comme indignes du beau nom de soldat, puisqu'ils violaient leur serment de fidélité " (trad. d'Ablancourt). Germanicus vient précisément d'employer lui-même le procédé avec ses soldats (An. I, 42, 2).

21. agréable aux soldats: An. I, 28, 3, «bonis artibus grati in vulgus» (nous reprenons la trad. d'Ablancourt, qui a «bonnes qualités»; trad. Burnouf: il jouissait "d'une popularité honnêtement acquise»). Le centurion a donc l'un des deux éléments posés, l'amitié ou benevolentia du peuple, à défaut de l'autorité ; autrement dit, son petit discours ne vaut pas grand'chose en lui-même. Clément a été auparavant le seul centurion épargné par la foule déchaînée, laquelle l'a gardé comme porte-parole pour transmettre les revendications des soldats (An. I, 23, 4). Tacite motive le fait par son « esprit assez vif », mais on comprend sans peine que c'était un officier populaire auprès de la troupe.

22. «1. Aen. " (note de Vossius)

23. "Suetonius in Caesare cap. 70. et Tacitus lib. 1. Annal. in Germanici oratione. Item Dio. hist. lib. 42.» (note de Vossius)

24. «Lib. 1. Annal. » (note de Vossius)

25. Autorité : « protégé » rend praesidium, le « double bouclier » du § 6 (voir note 19). Il s'en déduit que l'innocentia a ici à voir avec les dispositions amicales ou benevolentia du peuple envers l'orateur : ce dernier n'a pas nui au peuple (in/nocens pour non nocere), lequel n'a donc rien à lui reprocher.

26. Insensiblement: lat. sensim, un des adverbes typiques de l'insinuatio ou exorde face à un auditoire hostile. À la fin de son § 4, Tacite a ajouté : «Le silence ou de légers murmures [murmure modico] accueillirent ces paroles ». L'orateur perçoit ainsi que la troupe reste modérée dans ses réactions, et qu'il peut du coup aller plus loin et plus fort.

27. Changer d'humeur: au latin, même flexit qu'au $\S 6$ pour César et son «Citoyens!» (voir note 20).

28. Le revirement des soldats: Vossius reprend à Tacite les formules pour le cortège $(A n . \mathrm{I}, 40,4)$, la honte et la pitié (I, 41, 2, «Pudor et miseratio ») et enfin la rivalité ou jalousie envers les habitants de Trièves (I, 41, 3, « sed nihil aeque flexit quam invidia in Treviros »). Trad. d'Ablancourt : " On voit passer une troupe de femmes en un triste et misérable équipage; Agrippine comme fugitive portant son fils en son sein, les femmes des principaux Officiers tout éplorées et compagnes de sa fuite. »-Comme Vossius suit son idée qu'un discours n'est pas l'essentiel pour calmer une 
sédition, il ne cite pas le second et long discours de Germanicus, qui, en rebondissant sur la réaction de la troupe face au départ de sa femme, parachève le retour au calme (An. I, 42-43). Lisant et recopiant lui-même Vossius, Pelletier, lui, cite et analyse ce deuxième discours. On tient ainsi une sorte de conclusion.

D’une part, Pelletier en vient à reléguer hors champ la conciliatio 1 seule décrite par Érasme, en considérant qu'elle relève de la sphère privée : «De privata Conciliatione, qua nobis amorem alicujus conciliamus, nihil habeo quod dicam» (p.815, fin de son point IX). Cette formule de prétérition introduit un bref résumé, évoquant les § 3-4 de Vossius. De 1521 (Érasme) à 1641 (Pelletier), on a ainsi une évolution nette. L'idée irénique de la conciliatio 1 a cédé la place à une vision du monde moins pacifiée. Entre troubles divers des XVI ${ }^{\mathrm{e}}-\mathrm{XVII}{ }^{\mathrm{e}}$ siècles et succès du tacitisme, la guerre civile est devenu un thème majeur en Europe, d'où l'urgence de penser la réconciliation. D'autre part, Pelletier comme Vossius attribue un rôle fondamental au triste cortège des femmes. Vossius respecte l'enchaînement des faits chez Tacite, puisque ce cortège suit le premier et bref discours de Germanicus, dont l'analyse clôt son descriptif de la conciliatio 2. Mais Vossius pour ainsi dire triche avec Tacite, en ne mentionnant pas le deuxième et long discours de Germanicus. De ce discours long, Pelletier, lui, donne l'analyse, en partie décalquée de Vossius: Germanicus de nouveau « rappelle les triomphes et les victoires de Tibère, pour leur reprocher leur attitude présente avec un ton d'autant plus sévère et direct» (même severius qu'au $\S 7$ de Vossius). Pelletier voit là le «spécimen [specimen] remarquable d'un discours de Réconciliation ». Mais lui aussi triche avec Tacite. Chez l'historien, c'est bien le second discours qui achève de convaincre les soldats : " [An. I, 44, 1] Touchés par ce discours, ils lui demandent grâce, et, reconnaissant la vérité de ses reproches [exprobrari], ils le conjurent de punir le crime, de pardonner à l'erreur, et de les mener à l'ennemi. » Pelletier ne dit mot de cette fin.

Sa fidélité à Vossius l'entraîne à une conclusion étonnante : «Assurément, ce fut là un discours éloquent, par un orateur jouissant d'une autorité considérable, et pourtant cela ne suffit pas à calmer complètement l'émeute. Finalement, ce qui y parvint, ce fut le sens de l'honneur et la pitié, d'une part, et d'autre part la rivalité avec les Trévires [reprise littérale des formules de Vossius], auprès desquels son épouse partait se réfugier, portant sur son sein leur fils encore tout petit - voilà ce qui vainquit des esprits devenus sauvages ». Le triste cortège des femmes serait ainsi la clé ultime de l'efficacité. L'image spectaculaire aurait plus de pouvoir que les mots.

En bon jésuite, Pelletier valorise la douceur sur la violence, comme l'indique sa dernière formule (commentant An. I, 40, 4 à 41, 1) : «Voyez ce que peuvent ces remèdes plus tendres, bien que lents, contre des barbares et des sauvages!» Mais il esquive ainsi, comme Vossius, la violence déchaînée qui fait suite au deuxième discours de Germanicus. Car la troupe signe son retour en grâce en exécutant spontanément les principaux meneurs de la sédition. Vossius aurait pu trouver la même conclusion sanglante, après une conciliatio entre ennemis, chez Buchanan historien de l'Écosse, à partir de Junius (Orationum, I, p. 151-154). Le chancelier et le régent du royaume se livrent vers 1430 une guerre sans merci, ils n'ont jamais été alliés, et pourtant l'un des deux prend l'initiative de proposer à l'autre une alliance. Leur union faisant leur force, le tout se solde par l'assassinat assumé du clan Douglas, la troisième puissance qui règne sur l'Écosse. Le début des Annales de Tacite raconte une histoire tout aussi shakespearienne. La rude leçon des historiens est que s'allier aux uns, c'est ne plus être l'allié des autres. Mais il ne faut pas de sang sur la scène théorique. Ni Vossius ni Pelletier n'imaginent une conciliatio 3 qui serait une création et non un retour au statu quo ante : le passage à une nouvelle alliance, au prix du sang des alliés précédents. S'ils ont introduit la guerre, dont ne parle pas Érasme, comme lui ils restent obsédés par la paix.

29. « Lib. 1. Annal. » (note de Vossius) 


\section{AUTEURS}

\section{G. J. vossius}

Gerhard Johann Voss, 1577-1649 